\title{
Potentially avoidable Inter-Facility transfer from Veterans Health Administration emergency departments: A cohort study
}

Nicholas M. Mohr ${ }^{1,2,3^{*}}$ D, Chaorong Wu ${ }^{4}$, Michael J. Ward ${ }^{5,6}$, Candace D. McNaughton ${ }^{5,6}$, Kelly Richardson ${ }^{1}$ and Peter J. Kaboli, ${ }^{1,7}$

\begin{abstract}
Background: Inter-facility transfer is an important strategy for improving access to specialized health services, but transfers are complicated by over-triage, under-triage, travel burdens, and costs. The purpose of this study is to describe ED-based inter-facility transfer practices within the Veterans Health Administration (VHA) and to estimate the proportion of potentially avoidable transfers.

Methods: This observational cohort study included all patients treated in VHA EDs between 2012 and 2014 who were transferred to another VHA hospital. Potentially avoidable transfers were defined as patients who were either discharged from the receiving ED or admitted to the receiving hospital for $\leq 1$ day without having an invasive procedure performed. We conducted facility- and diagnosis-level analyses to identify subgroups of patients for whom potentially avoidable transfers had increased prevalence.

Results: Of 6,173,189 ED visits during the 3-year study period, 18,852 (0.3\%) were transferred from one VHA ED to another VHA facility. Rural residents were transferred three times as often as urban residents $(0.6 \%$ vs. $0.2 \%$, $p<0.001$ ), and $22.8 \%$ of all VHA-to-VHA transfers were potentially avoidable transfers. The 3 disease categories most commonly associated with inter-facility transfer were mental health (34\%), cardiac (12\%), and digestive diagnoses (9\%).

Conclusions: VHA inter-facility transfer is commonly performed for mental health and cardiac evaluation, particularly for patients in rural settings. The proportion that are potentially avoidable is small. Future work should focus on improving capabilities to provide specialty evaluation locally for these conditions, possibly using telehealth solutions.
\end{abstract}

Keywords: Emergency service, hospital, Regionalization, Rural health services, Hospitals, rural, Veterans health

\section{Background}

Over 2.4 million Veterans seek care from Veterans Health Administration (VHA) emergency departments (EDs) each year, and $40 \%$ of these Veterans live in rural America [1]. Providing high quality emergency care in low-volume centers is challenging, and several prior studies have suggested that clinical outcomes are worse

\footnotetext{
* Correspondence: nicholas-mohr@uiowa.edu

This work was presented at the National Update on Behavioral Emergencies conference (Las Vegas, NV) on December 14, 2018.

${ }^{1}$ Center for Comprehensive Access Delivery Research \& Evaluation (CADRE),

VA lowa City Healthcare System, lowa City, IA, USA

${ }^{2}$ Department of Emergency Medicine, University of lowa Carver College of Medicine, lowa City, USA

Full list of author information is available at the end of the article
}

in low-volume rural hospitals [2-9]. These volumeoutcome relationships could be attributable to provider training and experience, staffing, or resource allocation in low-volume facilities [10].

In many low-volume EDs, inter-facility transfer is used as a strategy for moving patients rapidly to hospitals equipped to care for them [11, 12]. Well-defined transfer networks have been developed for trauma and stroke care, but many patients with other conditions are transferred from EDs [11-14]. Both over-triage (transferring patients unlikely to benefit) and under-triage (failing to transfer patients likely to benefit) have been reported, and for some conditions the rate of potentially avoidable transfer (PAT) is high [15-18]. While $1.5 \%$ of US ED

(c) The Author(s). 2020 Open Access This article is distributed under the terms of the Creative Commons Attribution 4.0 International License (http://creativecommons.org/licenses/by/4.0/), which permits unrestricted use, distribution, and 
patients are transferred, that proportion can be much higher in rural hospitals, and access to specialists, technology, available inpatient capacity, and organizational factors can drive transfer practices [11].

The VHA provides critical emergency and specialty care to rural Veterans. All Veterans who present for care are evaluated and have standard diagnostic tests performed, but some that present to smaller facilities require transfer to larger facilities for diagnostic or therapeutic procedures or for availability of inpatient services. While inter-facility transfer provides access to care that would otherwise be unavailable in rural communities, it also imposes a substantial hardship for rural Veterans and their families by displacing them and increasing costs of care. Inter-facility emergency transfers often occur when Veterans are most vulnerable due to their acute illness, and complex care coordination can contribute to delays and triage mismatch $[12,19]$.

The objective of this study was to describe ED-based transfer patterns within in the VHA system, with a focus on potentially avoidable transfers (PAT). This analysis is the first step in developing targeted interventions such as ED-based telehealth to decrease the number of avoidable transfers and improve efficiency of inter-facility transfer within the VHA. To accomplish that objective, our goals were to (1) describe the VHA-to-VHA transfer population, (2) identify patient and health system factors associated with PAT, and (3) define geographic "hot spots" of high volumes of potentially avoidable transfer as a first step toward developing regional interventions. Our hypothesis was that geographic and diagnostic categories exist that are related to PATs, and that these transfers would disproportionately rural Veterans in smaller EDs on nights and weekends.

\section{Methods}

\section{Study design and setting}

This cohort study included all Veterans treated in VHA EDs and transferred to another VHA hospital between January 2012 and December 2014. This timeframe was selected to include only cases before implementation of International Classification of Diseases, 10th edition (ICD-10) in 2015. Data were abstracted from the VHA Clinical Data Warehouse $(\mathrm{CDW})$, which contains national data from clinical and administrative data systems collating patient-level, visit-level, provider-level, and institution-level information. This project was determined not to be human subjects research by the local Institutional Review Board (quality improvement), and this study is reported in accordance with the STrengthening the Reporting of OBservational Studies in Epidemiology (STROBE) statement [20].

\section{Selection of participants}

All adults who presented to one of the 120 VHA EDs were included in this study if they were transferred to another VHA acute care hospital. Patients presenting to urgent care clinics were excluded, and patients who were discharged to home, admitted locally, transferred to a non-VHA hospital, or who died in the ED were also excluded. Patients presenting by ambulance and diverted to a non-VHA facility were not included in this cohort. While some selection likely occurs in where Veterans receive emergency care, only patients who were treated in VHA EDs were included. Transferred patients were identified by linking the cohort of ED visits with all inpatient and ED visits (regardless of hospital) within $24 \mathrm{~h}$ after index ED arrival. Any patient who (1) had an inpatient or ED visit within $24 \mathrm{~h}$ at another VHA facility and (2) did not have a local hospital admission was considered to have been transferred to a VHA hospital. The 24-h visit window was permitted to allow for transfers that crossed midnight and therefore occurred on different dates.

\section{Facility-level variables}

Institutional variables were linked from the triennial VHA ED and Urgent Care Medical Director survey (collected in 2017) administered by the VHA Healthcare Analysis and Information Group (HAIG). This HAIG survey is administered to every medical director, with one response per facility (100\% response rate). ED visits were identified by inclusion in the Outpatient Data File, limited to Clinic Stop Code of 130 (emergency departments).

\section{Definitions}

For the purposes of this study, index hospital indicates the hospital of the first ED visit, and referral hospital indicates the destination hospital after inter-facility transfer. An ED transfer is a transfer with a referral hospital destination of an ED, and an inpatient transfer is a transfer with a destination of an inpatient service (either inpatient status or observation status) without an ED visit at the referral hospital. For the purposes of this study, inpatient and observation status were considered equivalent. Potentially avoidable transfers (PAT) were defined as transfers in which the patient was either discharged from the referral hospital ED or the patient was admitted to the referral hospital for less than $24 \mathrm{~h}$, discharged alive, and no procedures were coded. This definition is consistent with previous work in this area [17] and was intended to identify patients who were discharged quickly without a procedure that might have required specialty care. Specialist consultations were not considered to be procedures for the definition of PAT, because non-procedural opinions in many cases could 
be rendered without transfer. This definition was targeted to identify patients whose transfer may have been avoidable if real-time specialty telemedicine were available at index hospitals. The definition was not intended to suggest that PATs were inappropriate or could have been avoided with current resources. Non$V H A$ transfers were defined as having ED or inpatient visits reimbursed by the VHA to non-VHA hospitals within $24 \mathrm{~h}$ of the index ED visit (using VHA fee basis files), which occurs when VHA pays for care rendered for VHA-covered Veterans.

Primary discharge diagnosis was categorized based on the Clinical Classification Software (CCS) developed by the Agency for Healthcare Research and Quality (AHRQ) Health Care Utilization Project (HCUP), which groups primary ICD-9 diagnosis codes into mutually exclusive categories in a multi-level tiered system [21]. Procedures were defined by the HCUP Surgery Flag Software, which identifies invasive surgical procedures from Current Procedural Terminology (CPT) and ICD-9 procedure codes. For the purposes of this project, we used the broad definition of surgical procedures, which includes diagnostic procedures like cardiac catheterization and fiberoptic endoscopy if they are invasive, despite no therapy being performed. Rural Veterans were defined according to the address of residence, and classified according to Rural-Urban Commuting Areas (2 category approximation) [22]. Regional variation within the VHA was reported within Veteran Integrated Service Network (VISNs), a geographic classification whereby VHA facilities are organized into one of 21 regions. A follow-up visit within index or referral facilities was defined as an outpatient visit to any non-ED clinic within 45 days of hospital discharge to either the index hospital or referral hospital, respectively. All variables used in the analysis are included in Additional file 1.

\section{Estimate of transfer distances}

The driving distance between pairs of VHA hospitals for each transfer was calculated in miles. All geographic analysis and mapping was performed using ArcGIS v.10.6 (Environmental Systems Research Institute, Redlands, California).

\section{Outcomes}

The primary objective of this study was to identify common diagnoses, geographic regions, and health system factors associated PAT (primary outcome). Diagnoses were categorized into diagnosis group, and subcategories were examined to identify specific groups at highest risk of PAT. Secondary analyses included a temporal analysis of transfer patterns (e.g., day of the week, time of day), a description of the distance of transfer, and an estimate of the association between inter- facility transfer, follow-up visits, and mortality. Mortality was defined by using the date of death in the Veterans beneficiary record, it is was defined as death within 30 days.

\section{Analysis}

Inter-facility transfers were identified and then classified as potentially avoidable or not avoidable. Descriptive summary statistics describe the population of transferred patients overall and stratified by diagnosis, rurality, procedures performed after transfer, and geography. The unit of analysis was the ED visit.

Factors associated with PAT were identified using bivariate analysis (e.g., chi-squared for dichotomous predictors and t-test or Wilcoxon rank-sum test for continuous predictors, as appropriate). Because of the very large sample size, the investigators purposely selected factors based on a priori-defined hypotheses and where differences between the groups were clinically relevant, since statistical testing was able to identify very small and clinically irrelevant differences. Variables associated with PAT in bivariate analyses were included in an explanatory multivariable logistic regression model to identify the independent contribution of each of the constituent variables. All statistical tests are reported as two-tailed tests and were considered significant if $p<$ 0.05. All analyses were performed with SAS 9.4 (SAS Institute, Cary, NC). The sponsor did not contribute to the analytic plan or the reporting in any way.

\section{Results}

Over the 3-year study period, there were 6,173,189 VHA ED visits, of which $18,852(0.3 \%)$ were transferred to another VHA hospital (Fig. 1). The mean age of patients treated in the ED was 59 years, and $90 \%$ were male. VHA EDs treated a median of 15,989 patients annually (interquartile range 9895-22,341). Eighteen percent of the total cohort was admitted to the index VHA hospital. Rural Veterans (classified by home address) were at higher risk of VHA inter-facility transfer than urban Veterans $(0.6 \%$ vs. $0.2 \%, p<0.001)$. Of the total patients transferred from a VHA ED, 36\% were transferred to another VHA facility.

Of VHA transfers, $46 \%(n=8639)$ were transferred to another VHA ED, and the rest were transferred to another VHA facility inpatient unit. Median transfer distance was 81.5 miles (IQR 35.6-128.1 miles). PATs were identified in $22.8 \%(n=4292)$ of all VHA interfacility transfers. Of the total population with PATs, $74.6 \%(n=3355)$ were discharged from the referral ED, while the rest were discharged after a brief inpatient or observation stay. While 30-day mortality of transferred patients was low overall after VHA transfer $(n=487,2.6 \%)$, 65 patients died after being discharged with PAT (1.5\%). 


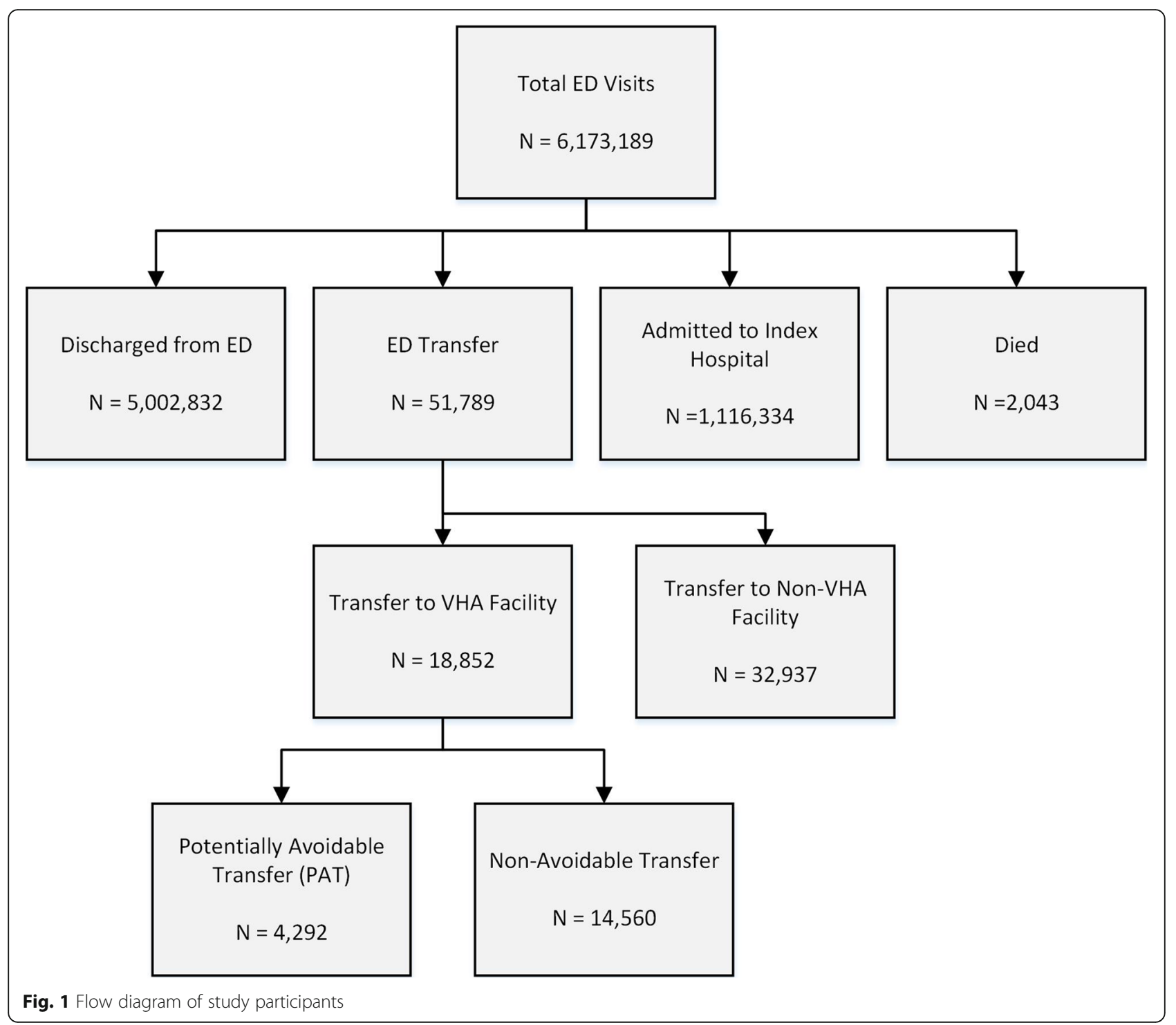

Factors associated with PAT include transfer to the referral ED (instead of inpatient unit), diagnosis, and location of transfer (Table 1). Although transfer overall was more common for rural Veterans, PAT was less prevalent $(20.8 \%$ for rural Veterans vs. $23.9 \%$ for urban Veterans, $p<0.001)$. PAT was more common during nights, evenings, and weekends compared with weekday transfers $(23.7 \%$ vs. $19.8 \%, \mathrm{p}<0.001)$, and the association between off-hours transfers and PAT was stronger for rural transfers than for urban transfers $(p=0.008)$.

\section{Primary transfer diagnoses}

The 3 diagnostic categories with most VHA transfers were mental health (CCS category 5, $n=6410$ [34\%]), cardiac (CCS category 7.2, $n=2161$ [12\%]), and digestive (CCS category 9, $n=1678$ [9\%]) conditions, with these top 3 categories comprising $55 \%$ of all transfers. The top
ICD-9 diagnosis related to VHA ED transfer was suicidal ideation (V62.84). Among patients transferred for a procedure, interventional cardiac procedures were most common, constituting $45 \%$ of all procedures (Additional file 2: Table S1). The distribution of diagnoses for Veterans who were transferred to non-VHA hospitals is similar to those transferred to VHA facilities, with the exception of transfers for circulatory conditions, which were more prevalent among non-VHA transfers (Fig. 2).

\section{Primary diagnoses related to potentially avoidable transfer}

Of all VHA transfers, the diagnostic categories associated with most PAT were mental health $(n=722$ [11\% potentially avoidable]) and cardiac $(n=452$ [21\% potentially avoidable]) diseases (Fig. 2). 
Table 1 Patient and hospital-level factors associated with VHAto-VHA ED inter-facility transfer, 2012-2014

\begin{tabular}{|c|c|c|c|}
\hline & $\begin{array}{l}\text { Non-Transfer } \\
(n=6,154,146)\end{array}$ & $\begin{array}{l}\text { Non-Avoidable } \\
\text { Transfer } \\
(n=14,560)\end{array}$ & $\begin{array}{l}\text { Potentially } \\
\text { Avoidable } \\
\text { Transfer } \\
(n=4292)\end{array}$ \\
\hline Age, y (SD) & $58.8(16.0)$ & $58.9(15.1)$ & $56.8(15.5)$ \\
\hline Male, n (\%) & $5,512,967(90)$ & $13,624(94)$ & $3930(92)$ \\
\hline \multicolumn{4}{|l|}{ Rurality of Residence } \\
\hline Urban, n (\%) & $5,078,808(89)$ & $9374(75)$ & $2899(78)$ \\
\hline Large Rural, n (\%) & $343,642(6)$ & $1678(13)$ & $420(11)$ \\
\hline Small Rural, n (\%) & $160,163(3)$ & $826(7)$ & $226(6)$ \\
\hline Isolated Rural, n (\%) & $135,530(2)$ & $653(5)$ & $177(5)$ \\
\hline \multicolumn{4}{|l|}{ Day of the Week } \\
\hline Monday, n (\%) & $1,050,212(17)$ & $2461(17)$ & $706(17)$ \\
\hline Tuesday, n (\%) & $978,139(16)$ & $2260(16)$ & $642(15)$ \\
\hline Wednesday, n (\%) & $934,969(15)$ & $2157(15)$ & $658(15)$ \\
\hline Thursday, n (\%) & $913,478(15)$ & $2131(15)$ & $682(16)$ \\
\hline Friday, n (\%) & $920,805(15)$ & $2171(15)$ & $552(13)$ \\
\hline Saturday, n (\%) & $679,584(11)$ & $1637(11)$ & $490(11)$ \\
\hline Sunday, n (\%) & $635,672(10)$ & $1743(12)$ & $562(13)$ \\
\hline \multicolumn{4}{|l|}{ Time of Day } \\
\hline 8a-5p Mon-Fri, n (\%) & $3,199,845(52)$ & $6998(48)$ & $1720(40)$ \\
\hline $\begin{array}{l}\text { Evenings, nights, and } \\
\text { weekends, } \mathrm{n}(\%)\end{array}$ & 2,913,014 (48) & $7562(52)$ & $2572(60)$ \\
\hline \multicolumn{4}{|l|}{ Transfer Location } \\
\hline ED, n (\%) & N/A & $5427(37)$ & $3212(75)$ \\
\hline Inpatient, n (\%) & N/A & $9133(63)$ & $1080(25)$ \\
\hline Hospitalization, n (\%) & $1,083,322(18)$ & $14,533(99)$ & 1435 (33) \\
\hline $\begin{array}{l}\text { Hospital Length of Stay, } d \\
\text { (median, IQR) }\end{array}$ & $4(2,7)$ & $5(3,9)$ & $1(1,1)$ \\
\hline Number of ED beds & $15.8(10.8)$ & $11.8(7.3)$ & $12.7(6.8)$ \\
\hline \multicolumn{4}{|l|}{ Follow-up care } \\
\hline $\begin{array}{l}\text { Visits at index hospital, } \\
\mathrm{n}(\%)\end{array}$ & $4,832,894(79)$ & $11,841(81)$ & $3139(73)$ \\
\hline $\begin{array}{l}\text { Visits at referral hospital, } \\
\text { n (\%) }\end{array}$ & N/A & $10,502(72)$ & $3266(76)$ \\
\hline 30-day Mortality, n (\%) & $82,259(1.3)$ & $422(2.9)$ & $65(1.5)$ \\
\hline
\end{tabular}

Abbreviations: $y$ years, $S D$ standard deviation, $E D$ emergency department, $d$ days, $I Q R$ interquartile range

\section{Health systems factors associated with potentially avoidable transfer}

Transferred Veterans were more commonly transferred from smaller index VHA EDs (12.1 vs. 15.8 beds, $p<$ 0.001 ), but smaller EDs did not have a higher prevalence of PAT (12.6 beds for PAT vs. 12.0 beds for non-PAT transfers, $p<0.001)$. That finding suggests that while smaller hospitals transfer a greater proportion of patients, ED size was not associated with transfer appropriateness. Hospitals staffed by at least $50 \%$ boardcertified emergency physicians had a lower proportion of VHA transfers than those that did not $(0.2 \%$ vs. $0.4 \%$, $p<0.001)$, but the proportion of PAT was higher in hospitals with more than $50 \%$ board-certified emergency physicians $(25 \%$ vs. $21 \%, p<0.001)$. Patients were more likely to be transferred from hospitals that did not accept incoming unscheduled ambulance traffic (e.g., 911 calls) into the ED ( $47 \%$ vs. $27 \%, p<0.001)$. For interpretation of computed tomography (CT) studies, transferring hospitals were more likely to use tele-radiology between 8 am and 5 pm Monday-Friday (8\% vs. 4\%) and were less likely to have radiology residents interpreting studies on nights and weekends (10\% vs. $21 \%)$, which is a proxy for the staffing and availability of specialized services at these facilities.

Patients who were transferred to other VHA hospitals were likely to follow-up within 45 days at the referral hospital (73\%), but they were equally likely to follow up at the index hospital compared with those who were not transferred (79\% vs. 79\%).

In summarizing data from the HAIG survey, $48 \%$ of medical directors of VHA EDs cited the transfer process as overly burdensome, with $29 \%$ identifying difficulties with identifying an accepting physician/facility, 13\% identifying challenges with obtaining approval for transfer, and over $65 \%$ noting that administrative processes contribute to delay in transfer.

\section{Geographic factors}

Variation in transfer proportions across index hospitals was high, ranging from 0 to $6 \%$ of total patients presenting to the ED who were transferred. PAT also varied widely, and PAT was not related to total transfer volume. Many high-transfer hospitals have an accompanying hospital that accepts the majority of transfers, but some sites have multiple transfer destinations (Fig. 3). There was also regional variation, with regional-level data showing transfer proportions that ranged from 8 to $53 \%$.

Although mental health-related transfers were the most prevalent condition for which VHA-to-VHA transfer was performed, in Veteran Integrated Service Network (VISN) numbers 7 (Alabama, Georgia, and South Carolina) and 17 (Texas), mental health transfers comprise over $60 \%$ of total inter-facility transfers. Similarly, circulatory system conditions comprise over $20 \%$ of total transfers VISN 1 (Connecticut, Maine, Massachusetts, Rhode Island, and Vermont), 6 (North Carolina and Virginia), and 15 (Kansas, Missouri, Southern Illinois and Indiana). Figure 4 shows VISNlevel variation in transfer volume and diagnosisspecific transfers. 


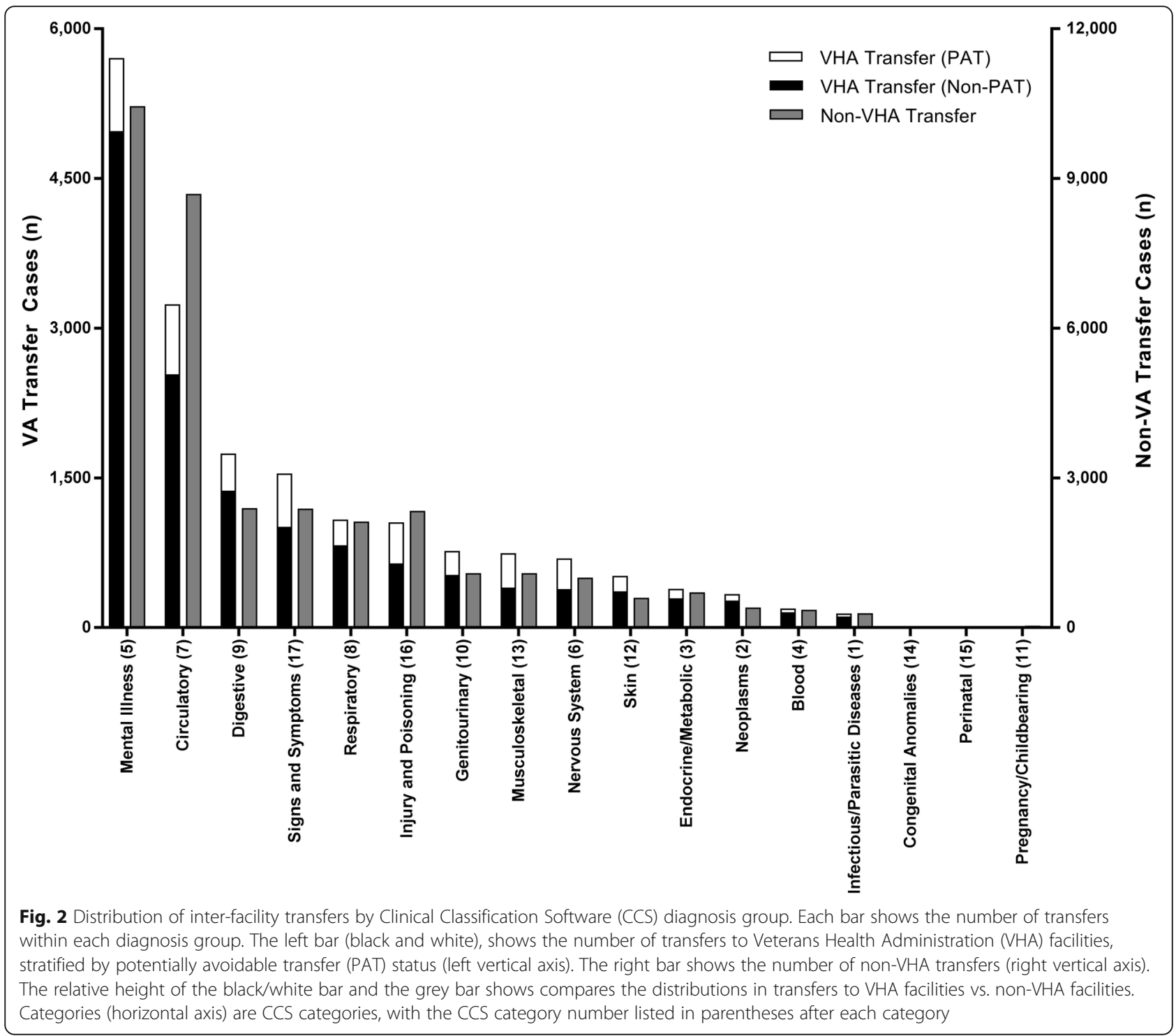

\section{Multivariable explanatory model}

Adjusting for factors associated with inter-facility transfer, ED arrival at the index hospital on nights and weekends (aOR 1.252) and hospitals with more than 50\% board-certified emergency physicians (aOR 1.266) both were associated with increased probability of PAT, while rural patients were at lower risk (aOR 0.798). The diagnostic categories associated with the highest adjusted risk were nervous system diseases (aOR 1.617), musculoskeletal conditions (aOR 1.571), and injury and poisoning (aOR 1.374). Importantly, however, the groups at highest risk did not constitute the greatest absolute number of PATs (Table 2).

\section{Mental health subgroup analysis}

Because of the high prevalence of mental health-related transfers, we conducted a post hoc subgroup analysis to identify factors associated with PAT within this group. The strongest risk factor for PAT (compared with both appropriate transfer and non-transfer) was being treated at night or on the weekend (67\% vs. $63 \%, p<$ 0.001). Characteristics of the subset of patients transferred for mental health are summarized in Additional file 3: Table S2.

\section{Discussion}

Our study identified several factors associated with inter-facility transfer, and mental health and cardiac disease are the two diagnosis groups for which inter-facility transfer is most prevalent in VHA hospitals. These findings highlight important differences between VHA healthcare and civilian healthcare systems, emphasizing the resources available within the VHA health system might be unique and underlining the need for VHA- 


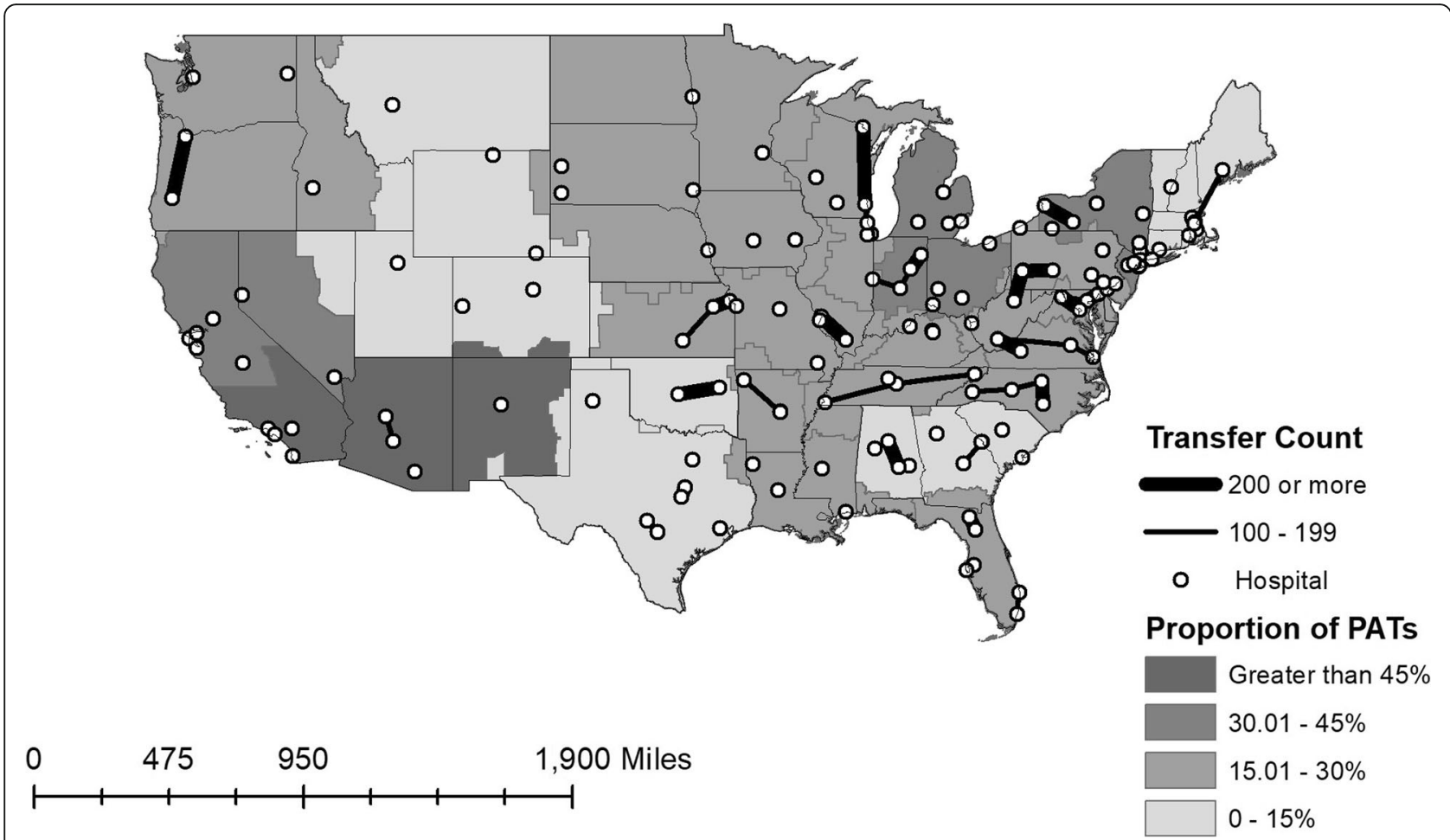

Fig. 3 Map of ED-based VHA-to-VHA inter-facility transfers, 2012-2014. Each dot on the map indicates a single VHA hospital with an emergency department (ED). Lines between these hospitals indicate transfers between facilities, with the thickness of the line represents the number of transfers. For some pairs of hospitals, the number of transfers are bidirectional, in which case the number of transfers in each direction are added together to represent the total transfer volume. Lines are not drawn between hospitals that have fewer than $100 \mathrm{VHA}$-to-VHA transfers over the study period. The proportion of transfers within each Veterans Integrated Service Network (VISN) that qualify as potentially avoidable transfers (PAT) is represented by grayscale shading (see legend). Note that non-VHA transfers are not included on this figure. The authors would like to acknowledge Morgan Swanson, BS for her assistance with preparation of this figure

specific solutions to health care delivery challenges. We also found that a sizeable portion of ED transfers from VHA hospitals refer patients outside the VHA.

The overall purpose of conducting this analysis was to identify areas where novel delivery of specialty care might avoid the need for some VHA transfers. In civilian health systems, ED-based telemedicine has been used to provide specialty provider and nursing support with the goal of improving transfer appropriateness [23-31]. This service has been most broadly implemented in telestroke care, providing real-time video consultation by a neurologist to an ED for the purpose of selecting patients for intravenous thrombolysis [32]. In some cases, inter-facility transfers have been avoided with targeted remote care [33-36] and allowed patients to remain near their families [37],which suggests that specialty telehealth capabilities may improve access, timeliness, and reduce the need for some emergency inter-facility transfers [23]. Some reports have even reported favorable provider-based outcomes related to telehealth implementation [38, 39].

Notably, over one-third of all VHA-to-VHA interfacility transfers are for patients with mental health diagnoses, higher than that reported in civilian hospitals. This prevalence could be related to a combination of (1) robust mental health resources available within the VHA and (2) limited local bed availability or high inpatient occupancy necessitating transfer for inpatient hospitalization. Compared with civilian hospitals, VHA facilities have fewer patients transferred with myocardial infarction, stroke, and traumatic injury [11].

The overall goal of this project was to identify populations within the VHA where unnecessary transfers could be avoided. From our data, mental health diagnoses represent a rich target population for which telehealth might offer a plausible solution. Importantly, we feel that targeting mental health transfers is important because of the size of the population, despite the fact that the raw risk of PAT among that population is not elevated. Mental health providers are in critical shortage in most of the US [40, 41], and patients with mental health crises often require emergency care [42]. Increasingly, specialty mental health evaluation is unavailable, especially during evenings and weekends [43, 44]. Real-time telemedicine has been used for psychiatric consultations, counseling, and ongoing care as a strategy to leverage limited 


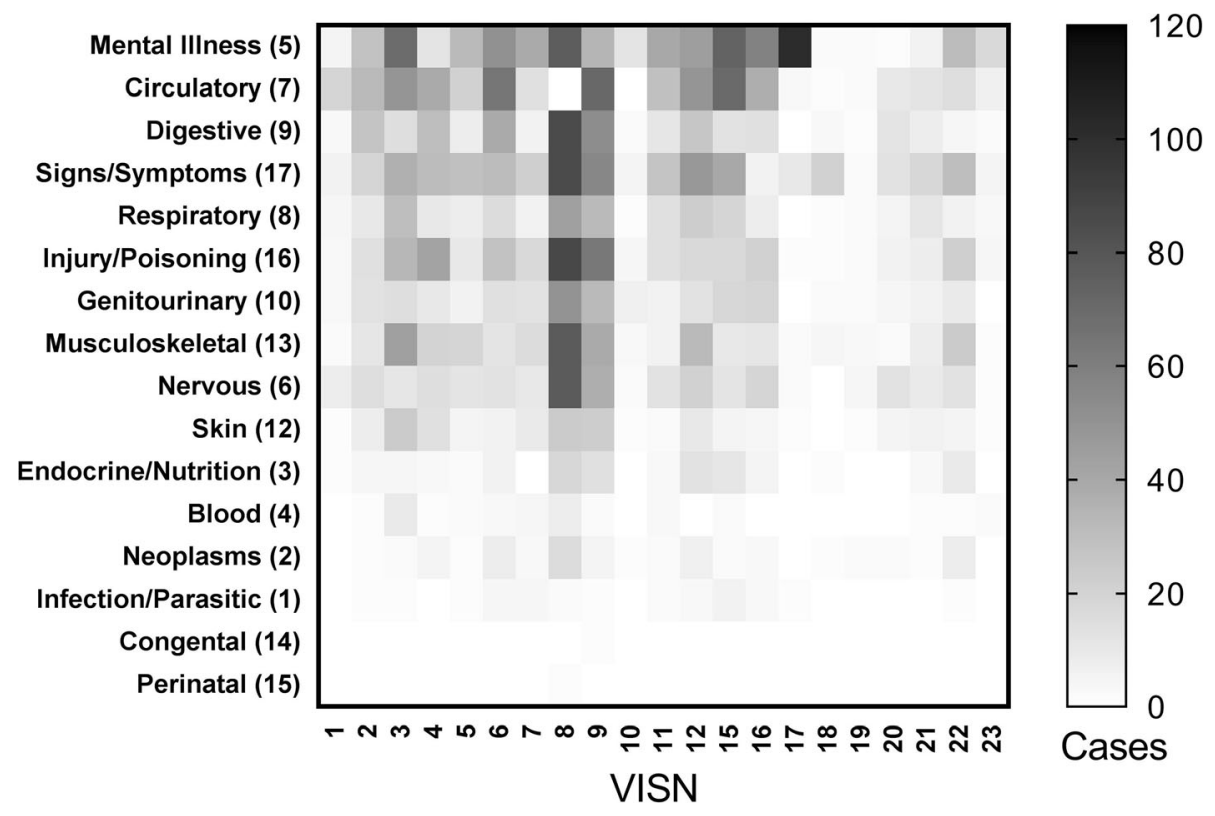

Fig. 4 Regional variation in potentially avoidable transfers (PATs) by Clinical Classification Software (CCS) diagnosis group. Each cell in the heat map represents the total number of potentially avoidable transfers within one Veterans Integrated Service Network (VISN) region. CCS diagnosis categories are listed on the vertical axis, with the diagnosis group number listed in parentheses after the CCS category abbreviation. VISN regions are listed on the horizontal axis. Darker colors represent more PATs within the VISN for the diagnosis group

psychiatric resources across geographic areas, and these networks have even provided care in emergency departments [40, 45-51]. Providing detailed psychiatric evaluation, treatment recommendations, and disposition guidance could be one important service that could improve cost-effective access to mental health professionals, especially for rural veterans. Emergency mental health care may be particularly amenable to telehealth interventions, whereas cardiac catheterization and gastrointestinal endoscopy may not.

While myocardial infarction, stroke, and digestive conditions represented many transfers and many could be potentially avoidable, these conditions also commonly require procedural capabilities, and the need for those procedures may not be immediately obvious. Future work could develop additional interventions to better target inter-facility transfer in the group of patients needing cardiac catheterization, stroke care, or endoscopy, but these populations remain relatively small. Future work could also partner with local EMS services to better guide prehospital diversion practices. Despite the enriched mental health transfer population within VHA facilities, patients with mental health emergencies may benefit from provider-to-provider telemedicine in civilian hospitals as well, because factors driving interfacility transfer and barriers to mental health access are ubiquitous outside the VHA.

A final important observation from this study surrounds the results from the HAIG survey of ED medical directors. Nearly half of medical directors of VHA EDs cited the transfer process as overly burdensome, and the majority noted that administrative processes resulted in transfer delays. While inter-facility transfer is complex, efforts to reduce the administrative burdens could be one effective way for patients to reach definitive care more quickly and reduce boarding in VHA EDs. In this study, we did not examine timeliness of transfer or the prevalence or impact transfer boarding has on VHA EDs, but the volume of transfers suggests that future work should examine the impact of these relationships in more detail.

Our study has several limitations. While administrative data provides a large sample, it limits the information available for individual patients to those coded in administrative claims. The second limitation is our definition of a transfer. The advantage of using the linkage method of identifying transfers is that we have high confidence that patients actually ended up receiving care in the receiving hospital. There could be some patients, however, who were discharged from the ED and presented to a different ED that we have inappropriately classified as transfer. Third, many patients were transferred from VHA EDs to non-VHA hospitals. While we were unable to assess PAT in non-VHA transfers, this remains an important population for future study. It also may be a population for which the motivations and outcome of interhospital transfer may differ from VHA transfers. Finally, our narrowly defined definition of PAT is not 
Table 2 Multivariable explanatory model for potentially avoidable transfer (PAT)

\begin{tabular}{|c|c|c|}
\hline Variable & Odds Ratio $(95 \% \mathrm{Cl})$ & $p$ \\
\hline Rural Residence & $0.798(0.715-0.890)$ & $<0.001$ \\
\hline $\begin{array}{l}\text { Arrival during Nights ( } 5 p-8 a) \\
\text { or Weekend }\end{array}$ & $1.252(1.144-1.370)$ & $<0.001$ \\
\hline Diagnosis Group (CCS) & & $<0.001$ \\
\hline Mental Illness (5) & $0.238(0.202-0.282)$ & \\
\hline Circulatory (7) & $0.518(0.437-0.615)$ & \\
\hline Digestive (9) & $0.563(0.464-0.683)$ & \\
\hline Signs and Symptoms (17) & 1.000 (ref) & \\
\hline Respiratory (8) & $0.568(0.456-0.708)$ & \\
\hline Injury and Poisoning (16) & $1.374(1.123-1.680)$ & \\
\hline Genitourinary (10) & $0.789(0.619-1.004)$ & \\
\hline Musculoskeletal (13) & $1.571(1.252-1.972)$ & \\
\hline Nervous System (6) & $1.617(1.288-2.030)$ & \\
\hline Skin (12) & $0.870(0.667-1.136)$ & \\
\hline Endocrine/Metabolic (3) & $0.535(0.380-0.752)$ & \\
\hline Neoplasms (2) & $0.470(0.321-0.690)$ & \\
\hline Blood (4) & $0.511(0.327-0.798)$ & \\
\hline Infectious/Parasitic Diseases (1) & $0.380(0.209-0.693)$ & \\
\hline VISN & & $<0.001$ \\
\hline 1 & $0.480(0.276-0.834)$ & \\
\hline 2 & $1.280(0.863-2.206)$ & \\
\hline 3 & $1.133(0.712-1.804)$ & \\
\hline 4 & $0.534(0.333-0.857)$ & \\
\hline 5 & $0.515(0.319-0.832)$ & \\
\hline 6 & $0.706(0.450-1.109)$ & \\
\hline 7 & $0.516(0.320-0.834)$ & \\
\hline 8 & $0.839(0.544-1.293)$ & \\
\hline 9 & $0.787(0.505-1.227)$ & \\
\hline 10 & $1.791(0.967-3.316)$ & \\
\hline 11 & $0.568(0.349-0.923)$ & \\
\hline 12 & $0.613(0.375-1.001)$ & \\
\hline 15 & $0.896(0.553-1.454)$ & \\
\hline 16 & $0.492(0.291-0.830)$ & \\
\hline 17 & $0.380(0.236-0.611)$ & \\
\hline 18 & $0.788(0.471-1.320)$ & \\
\hline 19 & $0.412(0.215-0.790)$ & \\
\hline 20 & $0.459(0.266-0.793)$ & \\
\hline 21 & $0.957(0.565-1.622)$ & \\
\hline 22 & $1.673(1.011-2.770)$ & \\
\hline 23 & 1.000 (ref) & \\
\hline $\begin{array}{l}\text { More than } 50 \% \text { Board-Certified } \\
\text { Emergency Physicians }\end{array}$ & $1.266(1.103-1.452)$ & $<0.001$ \\
\hline
\end{tabular}

Abbreviations: CCS Clinical Classification Software, VISN Veterans Integrated Service Network equivalent to avoidable transfer and some PAT may have still been necessary. Inter-facility transfer is a complex decision that would require more detailed qualitative patient-level analysis to better characterize specific reasons driving clinical decision-making.

\section{Conclusions}

In conclusion, inter-facility transfer occurs in $0.8 \%$ of VHA ED visits, and only one-quarter of these VHA-toVHA transfers are potentially avoidable. Rural Veterans are at high risk of VHA-to-VHA inter-facility transfer, but these transfers are no more likely to be PAT than for urban Veterans. Patients within VHA EDs are commonly transferred for mental illness, cardiac diseases, and digestive diseases, and there is wide variability between regions and hospitals in their transfer practices. Future work will focus on better understanding the reasons for transfers and factors that influence transfer decision-making, and future interventions will seek to improve the appropriateness, communication, and administrative factors surrounding inter-facility transfer.

\section{Supplementary information}

Supplementary information accompanies this paper at https://doi.org/10. 1186/s12913-020-4956-6.

Additional file 1. Data dictionary for variables included in analysis. Additional file 2: Table S1. Most common procedures performed after VHA-to-VHA inter-facility ED transfer.

Additional file 3: Table S2. Patient and hospital-level factors associated with VHA-to-VHA ED inter-facility transfer for mental health patients, 2012-2014.

\section{Abbreviations}

AHRQ: Agency for Healthcare Research and Quality; CCS: Clinical Classification Software; CDW: Clinical data warehouse; CPT: Current Procedural Terminology; CT: Computed tomography; ED: Emergency department; HAIG: Healthcare Analysis and Information Group; HCUP: Health Care Utilization Project; IQR: Inter-quartile range; PAT: Potentially avoidable transfer; STROBE: STrengthening the Reporting of OBservational Studies in Epidemiology; VHA: Veterans' Health Administration; VISN: Veteran Integrated Service Network

\section{Acknowledgments}

The authors would like to acknowledge Morgan Swanson, BS for her assistance with geographic analysis and the preparation of the map (Fig. 3). The work reported here was supported by the Department of Veterans Affairs, Veterans Health Administration, Office of Rural Health. The views expressed in this article are those of the authors and do not necessarily represent the views of the funding sources or the United States Government.

\section{Authors' contributions}

NMM, MJW, CDM, and PJK conceived the study, obtained funding, interpreted the findings, and drafted or critically revised portions of the manuscript. CW and KR conducted the analysis, contributed to interpretation of the findings, and critically revised the manuscript. All authors read and approved the final manuscript.

\section{Funding}

This material is based upon work supported by the Department of Veterans Affairs, Veterans Health Administration, VA Office of Rural Health (ORH-10708) 
and the Office of Research and Development, Health Services Research and Development (HSR\&D) Service through the Comprehensive Access and Delivery Research and Evaluation (CADRE) Center (CIN 13-412). Dr. Mohr additionally received support from the Agency for Healthcare Research and Quality (K08HS025753) and the Rural Telehealth Research Center, funded by the Federal Office of Rural Health Policy (U1C-RH29074), Health Resources and Services Administration (HRSA). Dr. Ward additionally received support from the National, Heart, Lung, and Blood Institute (K23HL127130). Dr. McNaughton additionally received supported from the 2017 Heart Failure Society of America/Emergency Medicine Foundation Acute Heart Failure Young Investigator Award funded by Novartis, the National Institutes of Health (K23-HL125670), and the Department of Defense (W81XWH-17-C-0252 from the CDMRP Defense Medical Research and Development Program). These contents are solely the responsibility of the authors and do not necessarily reflect the views of the United States Government or any of the funding sources. None of the funding agencies had access to or influenced the data set, analysis, or interpretation of the findings.

\section{Availability of data and materials}

The data that support the findings of this study are available from the Veterans Health Administration, but restrictions apply to the availability of these data, which were used under license for the current study and so are not publicly available. Data are, however, available from the authors upon reasonable request and with permission of the Veterans Health Administration.

\section{Ethics approval and consent to participate}

This project was determined not to be human subjects research by the University of lowa Institutional Review Board IRB-03.

\section{Consent for publication}

No consent for publication is required.

\section{Competing interests}

The authors declare that they have no competing interests.

\section{Author details}

${ }^{1}$ Center for Comprehensive Access Delivery Research \& Evaluation (CADRE), VA lowa City Healthcare System, lowa City, IA, USA. ${ }^{2}$ Department of Emergency Medicine, University of lowa Carver College of Medicine, lowa City, USA. ${ }^{3}$ Department of Anesthesia, University of lowa Carver College of Medicine, 200 Hawkins Drive, 1008 RCP, lowa City, IA 52242, USA. ${ }^{4}$ Institute for Clinical and Translational Sciences, University of lowa, lowa City, lowa, USA. ${ }^{5}$ Tennessee Valley Healthcare System VA Medical Center, Nashville, Tennessee, USA. 'Department of Emergency Medicine, Vanderbilt University Medical Center, Nashville, USA. ${ }^{7}$ Department of Internal Medicine, University of lowa Carver College of Medicine, lowa City, lowa, USA.

\section{Received: 10 June 2019 Accepted: 4 February 2020}

\section{We:}

\section{References}

1. Kaboli P. VAMC facility Rurality; 2011.

2. Kocher KE, Haggins AN, Sabbatini AK, Sauser K, Sharp AL. Emergency Department Hospitalization Volume and Mortality in the United States. Ann Emerg Med. 2014;64(5):446-57 e6.

3. Elmer J, Callaway CW, Chang CH, Madaras J, Martin-Gill C, Nawrocki P, et al. Long-Term Outcomes of Out-of-Hospital Cardiac Arrest Care at Regionalized Centers. Ann Emerg Med. 2018.

4. Fleet $R$, Bussières $S$, Tounkara FK, Turcotte $S$, Légaré F, Plant J, et al. Rural versus urban academic hospital mortality following stroke in Canada. PLoS One. 2018;13(1):e0191151.

5. Gonzales S, Mullen MT, Skolarus L, Thibault DP, Udoeyo U, Willis AW. Progressive rural-urban disparity in acute stroke care. Neurology. 2017; 88(5):441-8.

6. Leira EC, Hess DC, Torner JC, Adams HP. Rural-urban differences in acute stroke management practices. Arch Neurol. 2008;65(7):887-91.

7. Mann NC, Mullins RJ, Hedges JR, Rowland D, Arthur M, Zechnich AD. Mortality among seriously injured patients treated in remote rural trauma centers before and after implementation of a statewide trauma system. Med Care. 2001;39(7):643-53.
8. Masterson S, Wright P, O'Donnell C, Vellinga A, Murphy AW, Hennelly D, et al. Urban and rural differences in out-of-hospital cardiac arrest in Ireland. Resuscitation. 2015;91:42-7.

9. Vukmir RB. The influence of urban, suburban, or rural locale on survival from refractory prehospital cardiac arrest. Am J Emerg Med. 2004;22(2):90-3.

10. Hartley D. Rural health disparities, population health, and rural culture. Am J Public Health. 2004;94(10):1675-8.

11. Kindermann S, Mutter R, Pines J. Emergency Department Transfers to Acute Care Facilities, 2009, HCUP Statistical Brief \#155. Rockville, MD: Agency for Healthcare Research and Quality; 2013.

12. Feazel $L$, Schlichting $A B$, Bell GR, Shane DM, Ahmed A, Faine B, et al. Achieving regionalization through rural interhospital transfer. Am J Emerg Med. 2015;33(9):1288-96

13. Soto JM, Zhang Y, Huang JH, Feng D-X. An overview of the American trauma system. Chin J Traumatol. 2018;21(2):77-9.

14. Gorelick PB. Primary and comprehensive stroke centers: history, value and certification criteria. J Stroke. 2013;15(2):78-89.

15. Newgard CD, Staudenmayer K, Hsia RY, Mann NC, Bulger EM, Holmes JF, et al. The cost of overtriage: more than one-third of low-risk injured patients were taken to major trauma centers. Health Aff (Project Hope). 2013;32(9):1591-9.

16. Xiang H, Wheeler KK, Groner Jl, Shi J, Haley KJ. Undertriage of major trauma patients in the US emergency departments. Am J Emerg Med. 2014;32(9):997-1004.

17. Mohr NM, Harland KK, Shane DM, Miller SL, Torner JC. Potentially avoidable pediatric Interfacility transfer is a costly burden for rural families: a cohort study. Acad Emerg Med Off J Soc Acad Emerg Med. 2016;23(8):885-94.

18. Sorensen MJ, von Recklinghausen FM, Fulton G, Burchard KW. Secondary overtriage: the burden of unnecessary interfacility transfers in a rural trauma system. JAMA Surg. 2013;148(8):763-8.

19. O'Neill B, Parkinson L, Dwyer T, Reid-Searl K. Nursing home nurses' perceptions of emergency transfers from nursing homes to hospital: a review of qualitative studies using systematic methods. Geriatr Nurs. 2015; 36(6):423-30.

20. Ev E, Altman DG, Egger M, Pocock SJ, Gøtzsche PC, Vandenbroucke JP. Strengthening the reporting of observational studies in epidemiology (STROBE) statement: guidelines for reporting observational studies. BMJ. 2007:335(7624):806-8.

21. Healthcare Cost and Utilization Project (HCUP) Clinical Classification Software (CCS) Rockville, MD: Agency for Healthcare Research and Quality (AHRQ); 2017 [2017 Nov 10].

22. ZIP Code RUCA Approximation Seattle, Washington: WWAMI RUCA Rural Health Research Center; 2016 [Available from: http://depts.washington.edu/ uwruca/ruca-approx.php. Accessed 10 Jan 2018.

23. Ward MM, Jaana M, Natafgi N. Systematic review of telemedicine applications in emergency rooms. Int J Med Inform. 2015;84(9):601-16.

24. Mackinney AC, Ward MM, Ullrich F, Ayyagari P, Bell AL, Mueller KJ. The business case for Tele-emergency. Telemed J E Health. 2015;21(12):1005-11.

25. Mohr NM, Harland KK, Chrischilles EA, Bell A, Shane DM, Ward MM. Emergency department telemedicine is used for more severely injured rural trauma patients, but does not decrease transfer: a cohort study. Acad Emerg Med Off J Soc Acad Emerg Med. 2017;24(2):177-85.

26. Mohr NM, Vakkalanka JP, Harland KK, Bell A, Skow B, Shane DM, et al. Telemedicine use decreases rural emergency department length of stay for transferred North Dakota trauma patients. Telemed J E Health. 2018; 24(3):194-202.

27. Mohr NM, Young T, Harland KK, Skow B, Wittrock A, Bell A, et al. Telemedicine is associated with faster diagnostic imaging in stroke patients: a cohort study. Telemed J E Health. 2018.

28. Mohr NM, Young T, Harland KK, Skow B, Wittrock A, Bell A, et al. Emergency department telemedicine shortens rural time-to-provider and emergency department transfer times. Telemed J E Health. 2018;24(8):582-93.

29. Mueller KJ, Potter AJ, MacKinney AC, Ward MM. Lessons from teleemergency: improving care quality and health outcomes by expanding support for rural care systems. Health Aff (Project Hope). 2014;33(2):228-34.

30. Natafgi N, Shane DM, Ullrich F, MacKinney AC, Bell A, Ward MM. Using teleemergency to avoid patient transfers in rural emergency departments: an assessment of costs and benefits. J Telemed Telecare. 2018;24(3):193-201.

31. Ward MM, Ullrich F, MacKinney AC, Bell AL, Shipp S, Mueller KJ. Teleemergency utilization: in what clinical situations is tele-emergency activated? J Telemed Telecare. 2016;22(1):25-31. 
32. Baratloo A, Rahimpour L, Abushouk Al, Safari S, Lee CW, Abdalvand A. Effects of Telestroke on thrombolysis times and outcomes: a meta-analysis. Prehosp Emerg Care. 2018;22(4):472-84.

33. Hicks LL, Boles KE, Hudson ST, Madsen RW, Kling B, Tracy J, et al. Using telemedicine to avoid transfer of rural emergency department patients. J Rural Health. 2001;17(3):220-8.

34. Mehta S, Botelho R, Fernandez F, Prudente M, Cavalcanti R, Cade J, et al. TCT-50 fiscal impact of avoiding unnecessary transfers and hospitalization in STEMI interventions- results from LATIN America telemedicine infarct network (LATIN). J Am Coll Cardiol. 2018;72(13 Supplement):B22-B3.

35. Moya M, Valdez J, Yonas H, Alverson DC. The impact of a telehealth webbased solution on neurosurgery triage and consultation. Telemed J E Health. 2010;16(9):945-9.

36. Angileri FF, Cardali S, Conti A, Raffa G, Tomasello F. Telemedicineassisted treatment of patients with intracerebral hemorrhage. Neurosurg Focus. 2012;32(4):E6.

37. Mohr NM, Wong TS, Faine B, Schlichting A, Noack J, Ahmed A. Discordance between patient and clinician experiences and priorities in rural Interhospital transfer: a mixed methods study. J Rural Health. 2016; 32(1):25-34.

38. Reliford A, Adebanjo B. Use of Telepsychiatry in pediatric emergency room to decrease length of stay for psychiatric patients, improve resident on-call burden, and reduce factors related to physician burnout. Telemed J E Health. 2019;25(9):828-32.

39. Potter AJ, Mueller KJ, Mackinney AC, Ward MM. Effect of tele-emergency services on recruitment and retention of US rural physicians. Rural Remote Health. 2014;14(3):2787.

40. Meyer JD, McKean AJS, Blegen RN, Demaerschalk BM. Emergency department Telepsychiatry service model for a rural regional health system: the first steps. Telemed J E Health. 2018.

41. The Silent Shortage: A White Paper Examining Supply, Demand and Recruitment Trends in Psychiatry. Dallas, Texas: Merritt Hawkins; 2018.

42. Saba D, Levit K, Elixhauser A. Hospital Stays Related to Mental Health, 2006 Rockville, Maryland: Healthcare Cost and Utilization Project (HCUP) Statistical Briefs, Agency for Healthcare Research and Quality; 2008. p. 1-13.

43. Chihara I, Ae R, Kudo Y, Uehara R, Makino N, Matsubara Y, et al. Suicidal patients presenting to secondary and tertiary emergency departments and referral to a psychiatrist: a population-based descriptive study from Japan. BMC Psychiatry. 2018;18(1):112.

44. Bishop TF, Press MJ, Keyhani S, Pincus H. Acceptance of insurance by psychiatrists and the implications for access to mental health care. JAMA Psychiatry. 2014;71(2):176-81.

45. Roberts N, Hu T, Axas N, Repetti L. Child and adolescent emergency and urgent mental health delivery through Telepsychiatry: 12-month prospective study. Telemed J E Health. 2017;23(10):842-6.

46. Rachal J, Sparks W, Zazzaro C, Blackwell T. Highlight in Telepsychiatry and behavioral health emergencies. Psychiatric Clin North Am. 2017; 40(3):585-96

47. Saurman E, Lyle D, Kirby S, Roberts R. Use of a mental health emergency care-rural access programme in emergency departments. J Telemed Telecare. 2014;20(6):324-9.

48. Thomas JF, Novins DK, Hosokawa PW, Olson CA, Hunter D, Brent AS, et al. The use of Telepsychiatry to provide cost-efficient care during pediatric mental health emergencies. Psychiatr Serv. 2018;69(2):161-8.

49. Pangka KR, Chandrasena R, Wijeratne N, Mann M. Exploring the views of emergency department staff on the use of videoconferencing for mental health emergencies in southwestern Ontario. Stud Health Technol Inform. 2015;209:114-20

50. Yellowlees P, Burke MM, Marks SL, Hilty DM, Shore JH. Emergency telepsychiatry. J Telemed Telecare. 2008;14(6):277-81.

51. Chakrabarti S. Usefulness of telepsychiatry: a critical evaluation of videoconferencing-based approaches. World J Psychiatry. 2015;5(3):286-304.

\section{Publisher's Note}

Springer Nature remains neutral with regard to jurisdictional claims in published maps and institutional affiliations.

\section{Ready to submit your research? Choose BMC and benefit from}

- fast, convenient online submission

- thorough peer review by experienced researchers in your field

- rapid publication on acceptance

- support for research data, including large and complex data types

- gold Open Access which fosters wider collaboration and increased citations

- maximum visibility for your research: over $100 \mathrm{M}$ website views per year

At BMC, research is always in progress.

Learn more biomedcentral.com/submissions 